\title{
Multiscaling in passive scalar advection as stochastic shape dynamics
}

\author{
Omri Gat(1) and Reuven Zeitak(1)(2) \\ (1)Dept. Chem. Physics, The Weizmann Institute of Science, \\ Rehovot 76100, Israel \\ (2) Laboratoire de Physique Statistique, ENS, \\ 24 rue Lhomond, 75231 Paris Cedex 05, France
}

March 21, 2018

\begin{abstract}
The Kraichnan rapid advection model is recast as the stochastic dynamics of tracer trajectories. This framework replaces the random fields with a small set of stochastic ordinary differential equations. Multiscaling of correlation functions arises naturally as a consequence of the geometry described by the evolution of $N$ trajectories. Scaling exponents and scaling structures are interpreted as excited states of the evolution operator. The trajectories become nearly deterministic in high dimensions allowing for perturbation theory in this limit. We calculate perturbatively the anomalous exponent of the third and fourth order correlation functions. The fourth order result agrees with previous calculations.
\end{abstract}

\section{Introduction}

Although most researchers in the field of turbulence agree that the structure functions of the velocity field exhibit multiscaling, there is no well understood mechanism for this phenomenon which would allow, even in principle, a systematic calculation of the values of the multiscaling exponents [1]. The Kraichnan rapid advection model [2] is a simplified model for turbulent advection of a passive scalar in which a mechanism for multiscaling has been identified. Although the relevance of this mechanism to multiscaling in fully developed turbulence in still unclear, the study of multiscaling in Kraichnan's model presents a new approach and therefore has attracted a lot of interest

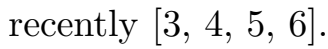

One of the main advances in studying this model was the identification of anomalous scaling contributions to correlation functions that are zero modes of certain linear 
partial differential operators [5]. Although this identification has allowed for the development of perturbation theories for the computation of the anomalous exponents, the meaning of these structures is, in our view, still somewhat obscure. The purpose of this paper is to reinterpret the anomalous exponents as eigenvalues of an evolution operator for the relative shape of trajectories of particles advected by the flow. The zero-modes arise naturally as the adjoints of the set of eigenfunctions of this evolution.

Our method is to consider the evolution of $N$ particles with the flow. These $N$ particles define a configuration that has an overall scale and a normalized shape. Under the flow dynamics, the overall scale tends to increase while the shape will asymptotically be described by a stationary measure. We consider the rate at which a distribution of initial shapes tends towards its stationary distribution. It is important to consider this rate in terms of the overall scale. Doing this we find the decay rate towards the stationary state to be a combination of power laws in this scale. We identify these power laws with the anomalous exponents of the passive scalar. Thus we view the anomalous scaling as a dynamic property of the evolution of trajectories [7].

This point of view treats the anomalous scaling as a geometric property of the trajectories of fluid particles in the flow, without explicit reference to the forcing. In fact, one does not need to define a passive scalar field since all the properties we are interested in are included in the shape dynamics. Note that this explains why the two point correlation functions do not have anomalous scaling - the geometry of a two point configuration is trivial, since it is completely described by the distance between the points.

We will show that this picture formally contains all the ingredients of anomalous scaling in the dynamics of passive scalars. Furthermore, it is useful as a calculational scheme. We will demonstrate this by presenting an alternative derivation for perturbation theory in large dimensions. We believe that the physics of passive scalar advection in large dimensions becomes more transparent in this framework. We calculate the previously unknown perturbative correction to the anomalous exponent of the third order correlation function, and recover the known result for the fourth order case [5].

In section 2, we present the path integral formalism of passive scalar advection, and show how correlation functions can be written as averages over Lagrangian trajectories. In section 3 we exploit this picture to identify a mechanism for generation of anomalous scaling, and show how the anomalous exponents are related to eigenvalues of an evolution operator. In section 4 we apply this picture to calculate perturbatively the first correction to the anomalous exponents in large dimensions. Section 5 is devoted to summary and conclusions.

\section{Lagrangian path formulation of passive scalar advection}

The dynamics of a passive scalar $\theta$ advected by an incompressible velocity field $\mathbf{u}$ are described by

$$
\partial_{t} \theta(\mathbf{r}, t)=\kappa \nabla^{2} \theta(\mathbf{r}, t)-\mathbf{u} \cdot \nabla \theta(\mathbf{r}, t)+f(\mathbf{r}, t)
$$

where $\kappa$ is the molecular diffusivity, and $f$ models the injection and extraction of scalar by external sources. The properties of $\mathbf{u}$ and $f$ are given, presumably as the result of some physical process.

In the rapid advection model [2] the velocity field is taken to be a random Gaussian 
field, delta correlated in time and self similar correlations in space:

$$
\left\langle\left(\mathbf{u}(\mathbf{r}, t)-\mathbf{u}\left(\mathbf{r}^{\prime}, t\right)\right) \otimes\left(\mathbf{u}\left(\mathbf{r}, t^{\prime}\right)-\mathbf{u}\left(\mathbf{r}^{\prime}, t^{\prime}\right)\right)\right\rangle=\mathbf{h}\left(\mathbf{r}-\mathbf{r}^{\prime}\right) \delta\left(t-t^{\prime}\right),
$$

where the "eddy-diffusivity" tensor $\mathbf{h}$ is defined by

$$
\mathbf{h}(\mathbf{r})=\left(\frac{r}{\ell}\right)^{\xi}\left(\mathbf{1}-\frac{\xi}{d-1+\xi} \frac{\mathbf{r} \otimes \mathbf{r}}{r^{2}}\right)
$$

where the coefficients are chosen such that $\nabla \cdot \mathbf{h}=0$. The forcing $f$ is also taken white in time, Gaussian, and is characterized by a single large scale $L$,

$$
\left\langle f(\mathbf{r}, t) f\left(\mathbf{r}^{\prime}, t^{\prime}\right)\right\rangle=\Xi\left(\mathbf{r}-\mathbf{r}^{\prime}\right) \delta\left(t-t^{\prime}\right),
$$

where the function $\Xi(r)$ is nearly constant for $r \ll L$ and decays rapidly for $r>L$.

The rapid advection model is theoretically attractive due to the fact that the equations for the correlation functions are linear partial differential equations as opposed to the hierarchy of nonlinear integro-differential equations for fully developed turbulence. Some theoretical progress has been made in the case of the rapid advection model and it is predicted that the $2 n$th order correlation function behaves as

$$
F_{2 n}\left(\mathbf{r}_{1}, \ldots, \mathbf{r}_{2 n}\right)=L^{n(2-\xi)}\left(c_{0}+\cdots+c_{k}(r / L)^{\zeta_{2 n}} \hat{F}_{2 n}(\hat{\mathbf{r}})+\cdots\right),
$$

where $r \ll L$ is a typical distance between points, and $\hat{\mathbf{r}}$ denotes a set of dimensionless variables describing the configuration of the $2 n$ points. According to the existing theory, the terms in the expansion ([) are of two types: Either they are inhomogeneous terms, whose scaling is determined by dimensional analysis, or they are the homogeneous solutions of moment equations, whose scaling exponents are not constrained by dimensional arguments. The exponents and scaling functions are expected to be universal, but not the $c$ coefficients, which depend on the details of forcing.

The exponent $\zeta_{2 n}$ which dominates when considering structure functions

$$
S_{2 n}\left(\mathbf{r}_{1}, \mathbf{r}_{2}\right)=<\left(\theta\left(\mathbf{r}_{1}, t\right)-\theta\left(\mathbf{r}_{2}, t\right)\right)^{2 n}>
$$

is the scaling of the leading term in (5) which depends non-trivially on all of the $2 n$ variables. Anomalous scaling occurs when this term is due to homogeneous solutions.

As the path integral formalism for equation (1) is well documented [8], we will not detail it here. The main conclusion is that one may write its solution formally

$$
\theta(\mathbf{r}, t)=\int_{-\infty}^{t} d t^{\prime}<f\left(\mathbf{r}^{\prime}\left(t^{\prime}\right), t^{\prime}\right)>_{\eta}
$$

with the trajectory $\mathbf{r}^{\prime}$ obeying

$$
\begin{aligned}
\mathbf{r}^{\prime}(t) & =\mathbf{r} \\
\partial_{t^{\prime}} \mathbf{r}^{\prime}\left(t^{\prime}\right) & =\mathbf{u}\left(\mathbf{r}^{\prime}\left(t^{\prime}\right), t^{\prime}\right)+\sqrt{2 \kappa} \eta\left(t^{\prime}\right)
\end{aligned}
$$

and $\eta$ is a vector of zero-mean independent Gaussian white random variables.

The physical meaning of this equation in simply understood if we consider the $\kappa=0$ case: the trajectories $y$ are simply the characteristics of the partial differential equation (PDE) (1), namely the Lagrangian trajectories of the flow field $\mathbf{u}$. If we want to know how much scalar is at point $(\mathbf{r}, t)$ we need to see what the value of $\theta$ was where this 
point came from, and add the accumulating forcing along the path traced backward in time [9]. When $\kappa$ is positive the trajectories are randomly perturbed Lagrangian trajectories and the scalar $\theta$ is obtained as an average over $\eta$. This procedure generates the diffusion term in equation (1).

Using the path integral formalism, we want to compute correlation function of the type

$$
F_{n}\left(\mathbf{r}_{1}, \ldots, \mathbf{r}_{n}\right)=\left\langle\theta\left(\mathbf{r}_{1}\right) \cdots \theta\left(\mathbf{r}_{n}\right)\right\rangle_{\mathbf{u}, f},
$$

where $\langle A\rangle_{\mathbf{u}, f}$ denotes an average over realizations of velocity and forcing. Substituting each factor of $\theta\left(\mathbf{r}_{i}\right)$ by its representation (7) we can perform the $f$ averages explicitly, yielding

$$
\begin{array}{r}
F_{2 n}\left(\mathbf{r}_{1}, \ldots, \mathbf{r}_{2 n}, t\right)=\left\langle\int _ { - \infty } ^ { t } d t _ { 1 } \cdots d t _ { n } \left[\Xi\left(\mathbf{r}_{1}^{\prime}\left(t_{1}\right)-\mathbf{r}_{2}^{\prime}\left(t_{1}\right)\right) \cdots\right.\right. \\
\left.\left.\times \Xi\left(\mathbf{r}_{2 n-1}^{\prime}\left(t_{n}\right)-\mathbf{r}_{2 n}^{\prime}\left(t_{n}\right)\right)+\text { permutations }\right]\right\rangle_{\mathbf{u}, \eta},
\end{array}
$$

while the odd moments vanish. Each of the trajectories $\mathbf{r}_{i}^{\prime}$ obeys an equation of the form (9), where $\mathbf{u}$ as well as $\eta$ is a stochastic variable, whose correlation function is given by (3). The procedure just described is a Langevin dynamics of $N$ particles, with correlated noise. Incompressibility of $\mathbf{u}$ implies that eq. (9) should be interpreted in the Ito sense. The corresponding Fokker-Planck equation with respect to both $\eta$ and $\mathbf{u}$ is precisely the well-known homogeneous Kraichnan PDE for the $2 n$th moment [10].

The representation of $F_{2 n}$ given in eq (11) can be turned into a recursive expression by ordering the $t_{1}, \cdots, t_{n}$ integrations such that $t_{1}>t_{2}>\cdots>t_{n}$. Since eq (11) contains all permutations in $\mathbf{r}_{k}^{\prime}$, this ordering can be done without changing the results. The times $t_{2}, \cdots, t_{n}$ can be integrated out to give

$$
\begin{aligned}
& F_{2 n}\left(\mathbf{r}_{1}, \ldots, \mathbf{r}_{2 n}, t\right)= \\
& \left\langle\int_{-\infty}^{t} d t_{1}\left[\Xi\left(\mathbf{r}_{1}^{\prime}\left(t_{1}\right)-\mathbf{r}_{2}^{\prime}\left(t_{1}\right)\right) F_{2 n-2}\left(\mathbf{r}_{3}^{\prime}\left(t_{1}\right), \cdots, \mathbf{r}_{2 n}^{\prime}\left(t_{1}\right)\right)+\text { permutations }\right]\right\rangle_{\mathbf{u}, \eta} .
\end{aligned}
$$

This form for $F_{2 n}$ is useful because it only contains a single time integration, so that one needs to follow a joint trajectory of $2 n$ particles at the same time and integrate along this trajectory the forcing defined by the terms in the square brackets in eq (12).

We intend to study the small $\kappa$ limit of the problem. If a given trajectory realization does not contain a 'near hit' among the trajectories, we expect that the $\kappa=0$ trajectories are in some sense near the small $\kappa$ trajectories. The only case where $\kappa=0$ differs radically from the small $\kappa$ limit is when two (or more) trajectories become closer than $O\left(\kappa^{\frac{1}{2-\xi}}\right)$. As we are interested only in separated correlation functions, we neglect the corrections to the $\kappa=0$ limit in what follows.

All the existing studies of the Kraichnan model are based on analyzing the Kraichnan linear PDEs. Most of these studies identify the anomalous contributions as scale invariant functions of the coordinates which are annihilated by the Kraichnan operator, thus they were termed zero modes. Most researchers accept the picture that correlation functions are obtained as a sum of zero modes with different anomalous scaling exponents and a contribution which is directly related to the forcing which is not anomalous. A notable exception to this approach is the Kraichnan closure, based on an appealing but unproven conjecture on conditional expectations of diffusive moments [3].

The common feature for these approaches is that the passive scalar is treated as a field. Here, we propose an alternative viewpoint based on eq. (11), in which all the 
properties of low order moments are extracted by looking at a small number of correlated stochastic trajectories. The representation (11) expresses $F_{2 n}$ as the expectation value of the forcing correlation function accumulated over the Lagrangian trajectories. Since the forcing drops off sharply beyond $L$ these objects measure the correlation of the times for which the trajectory positions are composed of pairs of points which are within distance $L$. In particular the two point moment $F_{2}$ is the average time during which two trajectories which end at given distance of each other stay within distance $L$. Excluding the case $d=2, \xi=0$ the trajectories will leave the vicinity of each other with probability 1 , and the integrals in (11) converge for each realization.

It should be noted that the representation described above can be used in principle as a numerical scheme for computing directly the correlation functions $F_{2 n}$ using a Monte-Carlo method without the need to generate the whole velocity field [11, 12].

In what follows we show how one can eliminate the forcing and the outer scale from the problem. Anomalous scaling arises from a relaxation process, in which the configuration tends towards an asymptotic stationary distribution, while the overall scale increases. The outer scale serves as a reference point for this process, but is not intrinsic to the process itself.

\section{Shape evolution operator and its relation to multiscal- ing}

A component of the evolution of an initial configuration is a rescaling of all the coordinates which all increase on the average like $t^{1 / \zeta_{2}}$; this rescaling is analogous to Richardson diffusion for the present case [1]. The dynamic exponent $\zeta_{2}=2-\xi$ is also the characteristic exponent of the second order structure function [2]. After factoring this overall expansion we are left with a normalized 'shape'. It is the evolution of this shape that determines the anomalous scaling.

Consider an initial shape $\mathbf{Z}_{0}$ with an overall scale $s_{0}$, that is, the configuration coordinates are $\mathbf{X}_{0}=s_{0} \mathbf{Z}_{0}$. Note that the shape coordinates are constrained. The overall scale is a homogeneous function of degree one of the coordinates $s_{0}=S\left(\mathbf{X}_{0}\right)$ but its exact functional form is to a large degree arbitrary.

The shape evolves in time. We fix a scale $s>s_{0}$ and examine the shape when the configuration reaches the scale $s$ for the first time (we know that this occurs in finite time with probability one). Since the trajectories are random the new shape $\mathbf{Z}$ is taken from a distribution $\gamma\left(\mathbf{Z}_{0}, \mathbf{Z}, \frac{s}{s_{0}}\right)$. We used scale invariance to deduce that the distribution is a function of $\frac{s}{s_{0}}$.

For a shape picked from an initial distribution of shapes $\rho_{s_{0}}\left(\mathbf{Z}_{0}\right)$ with scale $s_{0}$, the final shape distribution at scale $s$ is

$$
\rho_{s}(\mathbf{Z})=\int d \mathbf{Z}_{0} \rho_{0}\left(\mathbf{Z}_{0}\right) \gamma\left(\mathbf{Z}_{0}, \mathbf{Z}, \frac{s}{s_{0}}\right)
$$

For very large values of $\frac{s}{s_{0}}$ we expect the final shape distribution to approach an asymptotic distribution $\beta_{0}(\mathbf{Z})$. This distribution is invariant under the transformation (13) and is an eigenfunction of $\gamma$ with eigenvalue 1 . In general we expect a spectrum of eigenfunctions $\beta_{n}$ for $\gamma$ with eigenvalues $\alpha_{n}\left(\frac{s}{s_{0}}\right)$. By successive applications of $\gamma$ it follows that $\alpha_{n}\left(\frac{s}{s_{0}}\right)=\left(\frac{s}{s_{0}}\right)^{-\lambda_{n}}$. Furthermore we assume that the eigenfunctions $\beta_{n}(\mathbf{Z})$ 
form a complete set. Thus, any initial distribution of shapes $\rho_{s_{0}}(\mathbf{Z})$ can be written as

$$
\rho_{s_{0}}(\mathbf{Z})=\sum_{n} A_{n} \beta_{n}(\mathbf{Z})
$$

this distribution evolves into

$$
\rho_{s}(\mathbf{Z})=\sum_{m} A_{m}\left(\frac{s}{s_{0}}\right)^{-\lambda_{m}} \beta_{m}(\mathbf{Z}) .
$$

Consider an average of a correlation function $F_{n}(\mathbf{X})$ over $\rho_{s_{0}}$,

$$
\left\langle F_{n} \mid s_{0}\right\rangle=\int d \mathbf{Z} F_{n}(s \mathbf{Z}) \rho_{s_{0}}(\mathbf{Z})
$$

We now use the representation (12) for $F_{n}\left(s_{0}, \mathbf{Z}_{0}\right)$. We split the integration along the Lagrangian trajectory at the first point where the overall scale reaches the value $s$. It follows from the shape evolution equation (13) that we can write

$$
F_{n}\left(s_{0}, \mathbf{Z}_{0}\right)=I\left(s_{0}, s, \mathbf{Z}_{0}\right)+\int d \mathbf{Z} \gamma\left(\mathbf{Z}_{0}, \mathbf{Z}, \frac{s}{s_{0}}\right) F_{n}(s, \mathbf{Z}) .
$$

$I$ is the average of the contribution a specific forcing accumulated along the path from $s_{0}$ to $s$. The forcing is that of eq (12) so that for $s$ small enough its $L$ dependence is only through $F_{n-2}$. Averaging eq. (17) with $\rho_{s_{0}}\left(\mathbf{Z}_{0}\right)$ gives

$$
\int d \mathbf{Z}\left[\rho_{s_{0}}(\mathbf{Z}) F_{n}\left(s_{0} \mathbf{Z}\right)-\rho_{s}(\mathbf{Z}) F_{n}(s \mathbf{Z})\right]=\left\langle I\left(s_{0}, s\right)\right\rangle=\sum_{m} A_{m}\left(\bar{F}_{n}^{(m)}\left(s_{0}\right)-\left(\frac{s}{s_{0}}\right)^{\lambda_{m}} \bar{F}_{n}^{(m)}(s)\right) .
$$

Where

$$
\left.\bar{F}_{n}^{(m)}(s)\right)=\int \beta_{m}(\mathbf{Z}) F_{n}(s \mathbf{Z}) d \mathbf{Z}
$$

and

$$
\left\langle I\left(s_{0}, s\right)\right\rangle=\int \rho_{s_{0}}\left(\mathbf{Z}_{0}\right) I\left(s_{0}, s, \mathbf{Z}_{0}\right) d \mathbf{Z}_{0} .
$$

The average $\left\langle I\left(s_{0}, s\right)\right\rangle$ is dependent on $L$ only through the behavior $F_{n-2}$ with $L$. Thus, this term cannot have anomalous $L^{\left(\frac{n}{2}(2-\xi)-\zeta_{n}\right)}$ scaling. Since the $A_{m}$ 's are arbitrary, each term in the sum of equation (18) must be without anomalous $L^{\left(\frac{n}{2}(2-\xi)-\zeta_{n}\right)}$ scaling as well. The only way for this to occur is if the anomalous $L$-dependent part of $\left.\bar{F}_{n}^{(m)}(s)\right)$ is proportional to $f(L) s^{\lambda_{m}}$. Dimensional considerations imply that $f(L)$ also has a power-law behavior that fixes the dimension of $F_{n}$.

Thus, the projection of $F_{n}$ over $\beta_{m}$ selects a particular anomalous component. We conclude that the exponents $\lambda_{m}$, defined as the decay rates toward the invariant measure, are precisely the anomalous exponents of $F_{n}$.

We can use the eigenfunctions $\beta_{n}$ to expand the operator $\gamma$

$$
\gamma\left(\mathbf{Z}_{0}, \mathbf{Z}, \frac{s}{s_{0}}\right)=\sum_{m}\left(\frac{s}{s_{0}}\right)^{-\lambda_{m}} \beta_{m}(\mathbf{Z}) \mu_{m}\left(\mathbf{Z}_{0}\right) .
$$

$\gamma$ is a non-Hermitian operator, and therefore we can expect only that $\beta_{m}$ and $\mu_{m}$ are biorthogonal families of functions. This means that if we expand $F_{n}$ in terms of the 
functions $\mu_{m}$, a projection on $\beta_{m}$ will extract a single term of the sum, which has a pure anomalous scaling exponent. This leads us to identify the functions $\mu_{m}$ with the zero modes of the Kraichnan operator, which were previously identified in [5, 4] as the anomalous scaling structures.

We will use this formalism in the next section in an explicit perturbative calculation in large dimensions. This calculation demonstrates all the concepts discussed in this section, and shows how they can be used as a calculational method.

\section{Application: large $d$ perturbation theory}

The simplicity of the large dimension limit is due to the following reason: random trajectories tend to separate faster the larger the dimension. This is because there are more transversal directions in which to diffuse. This property, which is well known for Brownian motion, is also true for our case of correlated, Markovian paths. Therefore, in the limit of infinite dimensions the motion becomes a deterministic growth of the distance between each pair of points independently (note that the infinite number of directions available makes such a motion possible). This motion is simply the Richardson diffusion phenomenon, referred to above, in which relative distances $l$ increase like

$$
l \sim\left(l_{0}+t\right)^{1 /(2-\xi)} .
$$

This behaviour means that any initial $N$-point configuration will evolve towards a regular $N-1$ dimensional simplex, where all the relative distances are equal.

When $d$ is large but finite, trajectories fluctuate around the deterministic infinite dimensional limit (22), but the fluctuations are small, of order $O(1 / \sqrt{d})$. This forms the basis of perturbation theory in $1 / d$.

Our procedure will be to examine the expectation value of a non-dimensional symmetric combination of separations, which we denote $\sigma$. The relaxation of $\langle\sigma\rangle$ towards its asymptotic value is described by the set of relaxation exponents $\lambda_{m}$, see (21)

$$
\langle\sigma \mid s\rangle \equiv \int d \mathbf{Z} \sigma(\mathbf{Z}) \gamma\left(\mathbf{Z}_{0}, \mathbf{Z}, \frac{s}{s_{0}}\right)=\sum_{m}\left(\frac{s}{s_{0}}\right)^{-\lambda_{m}} \bar{\sigma}_{m} \mu_{m}\left(\mathbf{Z}_{0}\right)
$$

where $Z_{0}$ is the initial shape, implying an initial value for $\sigma\left(\mathbf{Z}_{0}\right)$. The $\bar{\sigma}_{m}$ 's defined as

$$
\int \bar{\sigma}_{m}=d \mathbf{Z} \sigma(\mathbf{Z}) \beta_{m}(\mathbf{Z})
$$

are numbers which only depend on the precise definition of $\sigma$. We see from (23) that the functional dependence of $\langle\sigma \mid s\rangle$ on the initial shape $\mathbf{Z}_{0}$ is given by the zero modes $\mu_{m}$. An asymptotic expansion in large $\frac{s}{s_{0}}$ will thus provide us with the leading zero modes, and their scaling exponents.

A term in the asymptotic expansion $\langle\sigma \mid s\rangle$ is expected to be, in the large $d$ limit, of the form

$$
\left(\frac{s}{s_{0}}\right)^{-\lambda^{(0)}-\frac{1}{d} \lambda^{(1)}+\cdots}\left[\mu^{(0)}\left(\mathbf{Z}_{0}\right)+\frac{1}{d} \mu^{(1)}\left(\mathbf{Z}_{0}\right)+\cdots\right] .
$$

Expanding the exponent gives

$$
\left(\frac{s}{s_{0}}\right)^{-\lambda^{(0)}}\left[\mu^{(0)}\left(\mathbf{Z}_{0}\right)+\frac{1}{d}\left(-\lambda^{(1)} \log \left(\frac{s}{s_{0}}\right)+\mu^{(1)}\left(\mathbf{Z}_{0}\right)\right)+\cdots\right] .
$$


Hence, the correction to the scaling exponent can be read off by looking at the loga-

rithmic term in $\frac{1}{d}$. Although nominally our expansion is in $\frac{1}{\sqrt{d}}$ it turns out that the first term vanishes (see below).

In principle, the statement that a logarithmic contribution is the first term in an expansion of a power needs to be justified by showing that all higher order terms of order $\frac{1}{d^{n}} \log \left(\frac{s}{s_{0}}\right)^{n}$ are consistent with the first term. In our case this is not necessary. Rather, we consider a perturbative expansion for the equivalent Fokker-Planck equation. In the Fokker-Planck equation the eigenvalues play the role of the exponents $\lambda_{m}$. Since perturbation theory finds corrections in the eigenvalues, we conclude that the logarithms must sum to a pure power as expected.

The perturbative analysis is going to carried out in terms of time as an independent variable rather than $s$. We thus need at some stage to transform to the $s$ variable in which our theory is formulated. Since the dynamics are nearly deterministic, for a given $s, t$ is very narrowly distributed around its Richardson diffusion value $t \sim s^{2-\xi}$. We show that to the order that was retained in the perturbation calculations it is sufficient to replace $t$ by its Richardson diffusion value.

\subsection{Representation in terms of separations, and perturbation theory}

The basic variables we use for analysis in high dimensions are the $n_{s} \equiv N(N-1) / 2$ inter-point square separations $\tilde{q}_{n m} \equiv\left(\mathbf{r}_{n}-\mathbf{r}_{m}\right)^{2}$. Their time evolution is obtained from (77) by the rules of the Ito calculus

$\frac{d \tilde{q}_{n m}(t)}{d t}=\left\langle\left(\mathbf{u}_{n}-\mathbf{u}_{m}\right)^{2}\right\rangle+2\left(\mathbf{r}_{n}-\mathbf{r}_{m}\right) \cdot\left(\mathbf{u}_{n}-\mathbf{u}_{m}\right)=2 \frac{\tilde{q}_{n m}(t)^{\xi / 2}}{\ell^{\xi}}\left(d-\frac{\xi}{d-1+\xi}\right)+\tilde{\eta}_{n m}(t)$,

where the second equality serves to define the noise term $\tilde{\eta}_{n m}$, a zero mean, $\delta$-correlated in time Gaussian process. The equations are again to be interpreted as Ito SDEs. The $n_{s}$ separations are subject to triangle inequalities which are preserved by the dynamics (27).

The deterministic part in eqs. (27) gives simple Richardson diffusion with the exponent $2 /(2-\xi) \equiv 2 / \zeta_{2}$, whereas all the non-trivial behavior is contained in the $\tilde{\eta}$ terms which couple between the different separations. When $d$ is large the noise term in (27) becomes small with respect to the deterministic term, and this phenomenon serves as the starting point for perturbation theory. In order to demonstrate this it is convenient to factor out the super-diffusive behavior from $\tilde{q}_{n m}$, defining

$$
\tilde{q}_{n m}(t)=\left(\frac{2-\xi}{\ell^{\xi}} d\left(t+\tau_{n m}\right)\right)^{2 /(2-\xi)} q_{n m}(t)
$$

where $\tau_{n m}$ is a constant determined by initial conditions. Substituting in eq. (27) yields (here and in the following we neglect the higher order term $\xi /(d-1+\xi)$ coming from incompressibility)

$$
\dot{q}_{n m}=\frac{2}{2-\xi} \frac{1}{t+\tau_{n m}}\left(q_{n m}^{\xi / 2}-q_{n m}\right)+\frac{1}{\sqrt{d}} \eta_{n m}(t),
$$

where

$$
\eta_{n m}=\left(\frac{2-\xi}{\ell^{\xi}} d\left(t+\tau_{n m}\right)\right)^{-2 /(2-\xi)} \tilde{\eta}_{n m}
$$


The covariance of $\eta_{n m}$ is (using the abbreviation $\left.\bar{q}_{n m}=\left(t+\tau_{n m}\right)^{2 /(2-\xi)} q_{n m}\right)$

$$
\begin{aligned}
&\left\langle\eta_{n m}(t) \eta_{k l}\left(t^{\prime}\right)\right\rangle=\frac{2}{\zeta_{2}}\left(t+\tau_{n m}\right)^{-2 / \zeta_{2}}\left(t+\tau_{n l}\right)^{-2 / \zeta_{2}} \\
& \times\left(\bar{q}_{n l}-\bar{q}_{m l}-\bar{q}_{n k}+\bar{q}_{m k}\right)\left(\bar{q}_{n l}^{1-\zeta_{2} / 2}-\bar{q}_{m l}^{1-\zeta_{2} / 2}-\bar{q}_{n k}^{1-\zeta_{2} / 2}+\bar{q}_{m k}^{1-\zeta_{2} / 2}\right) \delta\left(t-t^{\prime}\right) .
\end{aligned}
$$

This covariance is of order 1 in $d$, so that the stochastic term in eq. (29) is indeed small for $d \gg 1$. Thus, the dynamics of eq. (29) are dominated by the attractive fixed point $q_{n m}=1$ of the deterministic term, and the random term causes small fluctuations around it.

Perturbation theory is performed in a straightforward manner by expanding

$$
q_{n m}=1+\frac{1}{\sqrt{d}} q_{n m}^{(1)}+\frac{1}{d} q_{n m}^{(2)}+\ldots
$$

and

$$
\eta_{n m}=\frac{1}{\sqrt{d}} \eta_{n m}^{(1)}+\frac{1}{d} \sum_{k l} \eta_{n m, k l}^{(2)} q_{k l}^{(1)}+\ldots
$$

The noise terms are white Gaussian zero-mean processes. The correlation matrix of $\eta_{n m}^{(1)}$, is obtained from (31) by substituting $s_{n m} \rightarrow 1-\delta_{n m}$. The correlations involving $\eta_{n m}^{(2)}$ are more complicated, but are not needed to the order that we keep in the calculations.

The terms in the perturbation series (32) obey linear inhomogeneous equations:

$$
\dot{q}_{n m}^{(1)}=-\frac{1}{t+\tau_{n m}} q_{n m}^{(1)}+\eta_{n m}^{(1)},
$$

and

$$
\dot{q}_{n m}^{(2)}=-\frac{1}{t+\tau_{n m}}\left(q_{n m}^{(2)}+\frac{\xi}{4}\left(q_{n m}^{(1)}\right)^{2}\right)+\sum_{k l} \eta_{n m, k l}^{(2)} q_{k l}^{(1)},
$$

with respective solutions

$$
q_{n m}^{(1)}(t)=\frac{1}{t+\tau_{n m}} \int_{0}^{t} d t^{\prime}\left(t^{\prime}+\tau_{n m}\right) \eta_{n m}^{(1)}\left(t^{\prime}\right),
$$

and

$$
q_{n m}^{(2)}(t)=\frac{1}{t+\tau_{n m}} \int_{0}^{t} d t^{\prime}\left[-\frac{\xi}{4} q_{n m}^{(1)}(t)^{2}+\left(t^{\prime}+\tau_{n m}\right) \sum_{k l} \eta_{n m, k l}^{(2)}\left(t^{\prime}\right) q_{k l}^{(1)}\left(t^{\prime}\right)\right] .
$$

\subsection{Results for three-and four-point functions}

In this section we are going to use the results of perturbation theory derived above to demonstrate multiscaling in the three- and four-point correlation functions. For this purpose we make the following (arbitrary) choice

$$
\sigma \equiv n_{s} \frac{\sum_{n m} \tilde{q}_{n m}^{2}}{\left(\sum_{n m} \tilde{q}_{n m}\right)^{2}} .
$$

The definition of the overall scale is chosen to be

$$
s=\sqrt{\frac{1}{N} \sum_{n<m} \tilde{q}_{n m}} .
$$


It is shown in the following subsection that replacing $t$ by $s^{\zeta_{2}}$ is correct to the order which we keep in the following calculation.

Substituting the perturbation series (32) to order $1 / d$ gives the expansion $\sigma=$ $\sigma^{(0)}+\frac{1}{\sqrt{d}} \sigma^{(1)}+\frac{1}{d} \sigma^{(2)}+\cdots$, where we have defined

$$
\begin{aligned}
& \sigma^{(0)}=n_{s} \frac{\sum_{n m} \rho_{n m}^{4 / \zeta_{2}}}{\left(\sum_{n m} \rho_{n m}^{2 / \zeta_{2}}\right)^{2}} \\
& \sigma^{(1)}=\sigma^{(0)}\left[2 \frac{\sum_{n m} \rho_{n m}^{4 / \zeta_{2}} q_{n m}^{(1)}}{\sum_{n m} \rho_{n m}^{4 / \zeta_{2}}}-2 \frac{\sum_{n m} \rho_{n m}^{2 / \zeta_{2}} q_{n m}^{(1)}}{\sum_{n m} \rho_{n m}^{2 / \zeta_{2}}}\right] \\
& \sigma^{(2)}=\sigma^{(0)}\left[\frac{\sum_{n m} \rho_{n m}^{4 / \zeta_{2}}\left(q_{n m}^{(1)}\right)^{2}}{\sum_{n m} \rho_{n m}^{4 / \zeta_{2}}}+2\left(\frac{\sum_{n m} \rho_{n m}^{4 / \zeta_{2}} q_{n m}^{(2)}}{\sum_{n m} \rho_{n m}^{4 / \zeta_{2}}}-\frac{\sum_{n m} \rho_{n m}^{2 / \zeta_{2}} q_{n m}^{(2)}}{\sum_{n m} \rho_{n m}^{2 / \zeta_{2}}}\right)\right. \\
& \left.+3\left(\frac{\sum_{n m} \rho_{n m}^{2 / \zeta_{2}} q_{n m}^{(1)}}{\sum_{n m} \rho_{n m}^{2 / \zeta_{2}}}\right)^{2}-4 \frac{\sum_{n m} \rho_{n m}^{4 / \zeta_{2}} q_{n m}^{(1)}}{\sum_{n m} \rho_{n m}^{4 / \zeta_{2}}} \frac{\sum_{n m} \rho_{n m}^{2 / \zeta_{2}} q_{n m}^{(1)}}{\sum_{n m} \rho_{n m}^{2 / \zeta_{2}}}\right] .
\end{aligned}
$$

(We used the abbreviation $\rho_{n m}=t+\tau_{n m}$.)

When $t \gg \tau_{n m},\langle\sigma\rangle$ is dominated by contributions from the leading zero modes, and we therefore check its asymptotics in this limit. In the limiting case $d=\infty$ we have, for $t \rightarrow \infty$

$$
\begin{aligned}
& \langle\sigma\rangle=\sigma^{(0)} \sim n_{s} \frac{\sum_{n m} 1+\left(4 / \zeta_{2}\right)\left(\tau_{n m} / t\right)+\left(2 / \zeta_{2}\right)\left(4 / \zeta_{2}-1\right)\left(\tau_{n m} / t\right)^{2}}{\left(\sum_{n m} 1+\left(2 / \zeta_{2}\right)\left(\tau_{n m} / t\right)+\left(1 / \zeta_{2}\right)\left(2 / \zeta_{2}-1\right)\left(\tau_{n m} / t\right)^{2}\right)^{2}} \\
& \sim 1+\frac{4}{n_{s} \zeta_{2}^{2}} \sum_{n m}\left(\frac{\tau_{n m}}{t}\right)^{2}-\frac{4}{\left(n_{s} \zeta_{2}\right)^{2}}\left(\sum_{n m} \frac{\tau_{n m}}{t}\right)^{2} .
\end{aligned}
$$

In the special case $N=3, n_{s}=3$ eq. (43) can be written as

$$
\sigma^{(0)} \sim 1+\frac{8}{9 \zeta_{2}^{2}} \frac{Z_{3}}{t^{2}}
$$

where $Z_{3}$ is the leading three-point zero mode,

$$
Z_{3}=\sum_{\substack{n \neq m, l \\ m<l}}\left(\tau_{n m}-\tau_{n l}\right)^{2}
$$

$\sigma^{(0)}$ is thus a linear combination of zero modes (up to order $t^{-2}$ ) as expected.

The $O(1 / \sqrt{d})$ contribution $\left\langle\sigma^{(1)}\right\rangle$ vanishes since $\left\langle\eta^{(1)}\right\rangle=0$, so that we proceed to examine the $O(1 / d)$ term. Up to order $t^{-2},\langle\sigma\rangle$ contains, in addition to the constant term, a term which is proportional to $\log t / t^{2}$. This term reflects the dependence of the scaling exponent on $d$, and we will therefore display only this term explicitly.

The first step consists of calculating moments of the $s$ functions:

$$
\begin{array}{r}
\left\langle q_{n m}^{(1)}(t)^{2}\right\rangle=\frac{1}{\left(t+\tau_{n m}\right)^{2}} \int_{0}^{t} d t^{\prime} d t^{\prime \prime}\left(t^{\prime}+\tau_{n m}\right)\left(t^{\prime \prime}+\tau_{n m}\right)\left\langle\eta_{n m}^{(1)}(t) \eta_{n m}^{(1)}\left(t^{\prime}\right)\right\rangle \\
=\frac{4}{\zeta_{2}}\left(1-\frac{\tau_{n m}^{2}}{\left(t+\tau_{n m}\right)^{2}}\right) .
\end{array}
$$




$$
\left\langle q_{n m}^{(2)}(t)\right\rangle=\frac{1}{\left(t+\tau_{n m}\right)}\left(-\frac{\xi}{4}\right) \int_{0}^{t} d t\left\langle q_{n m}^{(1)}\left(t^{\prime}\right)^{2}\right\rangle=-\frac{2-\zeta_{2}}{\zeta_{2}} \int_{0}^{t} d t^{\prime}\left(1-\frac{\tau_{n m}^{2}}{\left(t^{\prime}+\tau_{n m}\right)^{2}}\right) .
$$

(The term proportional to $\eta^{(2)}$ drops in averaging due to the Ito convention). Thus, terms involving only one separation do not contribute logarithmic terms to this order.

The large $t$ asymptotics of the cross correlation of two separations with one common vertex is

$$
\begin{aligned}
& \left\langle q_{n m}^{(1)}(t) q_{n l}^{(1)}(t)\right\rangle \sim c_{0 n m l}+\frac{c_{1 n m l}}{t}+ \\
& \quad \frac{2}{\zeta_{2}} \frac{\log t}{t^{2}}\left(\frac{2}{\zeta_{2}}-1\right)\left[\frac{1}{\zeta_{2}}\left(\tau_{n m}^{2}+\tau_{n l}^{2}\right)+\left(\frac{2}{\zeta_{2}}-1\right)\left(\tau_{n m}^{2}+\tau_{n l}^{2}-\tau_{m l}^{2}\right)+\left(\frac{2}{\zeta_{2}}-1\right) \tau_{n m} \tau_{n l}\right. \\
& \left.\quad-\left(\frac{4}{\zeta_{2}}-1\right)\left(\tau_{n m}+\tau_{n l}\right)\left(\tau_{n m}+\tau_{n l}-\tau_{m l}\right)+\frac{2}{\zeta_{2}}\left(\tau_{n m}+\tau_{n l}-\tau_{m l}\right)^{2}\right] .
\end{aligned}
$$

Summing over permutations gives

$$
\sum_{\substack{n \neq m, l \\ m<l}}\left\langle q_{n m}^{(1)}(t) q_{n l}^{(1)}(t)\right\rangle \sim \tilde{c}_{0}+\frac{\tilde{c}_{1}}{t}+\frac{2}{\zeta_{2}} \frac{\log t}{t^{2}}\left(\frac{2}{\zeta_{2}}-1\right)\left(\frac{2}{\zeta_{2}}+1\right) Z_{3} .
$$

We conclude that the only terms in $\left\langle\sigma^{(2)}\right\rangle$ which generate logarithms are the ones involving cross correlations of $q$ variables, i.e., the last two in eq. (42). Of these terms we need only keep the leading order in $1 / t$, giving

$$
\left\langle\sigma^{(2)}\right\rangle=c_{0}+\frac{c_{1}}{t}-\frac{4}{9 \zeta_{2}}\left(\frac{2}{\zeta_{2}}-1\right)\left(\frac{2}{\zeta_{2}}+1\right) Z_{3} \frac{\log t}{t^{2}} .
$$

Combining the contributions from (44) and (50) yields

$$
\langle\sigma\rangle \sim 1+\frac{8}{9 \zeta_{2}^{2}} \frac{Z_{3}}{t^{2}}\left(1-\frac{\xi}{2}\left(\frac{2}{2-\xi}+1\right) \frac{\log t}{d}\right) .
$$

which implies, after substituting $t \rightarrow s^{2-\xi}$

$$
\zeta_{3}=2(2-\xi)+\frac{\xi}{2 d}(4-\xi)+O\left(\frac{1}{d^{3 / 2}}\right) .
$$

A similar analysis is needed for the case $N=4, n_{s}=6$. The infinite dimensional limit becomes in this case

$$
\sigma^{(0)} \sim 1+\frac{1}{9 \zeta_{2}^{2}} \frac{Z_{3}+Z_{4}}{t^{2}}
$$

where the leading order 4-point zero mode is

$$
Z_{4}=\sum_{\substack{n<m, k<l \\ n<k}}\left(\tau_{n m}-\tau_{k l}\right)^{2}
$$

We also need cross correlations of separation variables without a common vertex, whose large $t$ asymptotics are

$$
\left\langle q_{n m}^{(1)} q_{k l}^{(1)}\right\rangle \sim\left(\frac{2}{\zeta_{2}}\right)^{2}\left(\frac{2}{\zeta_{2}}-1\right) \frac{\log t}{t^{2}}\left(\tau_{n l}-\tau_{m l}-\tau_{n k}+\tau_{m k}\right)^{2},
$$


and summing over permutations gives

$$
\sum_{\substack{n<m, k<l \\ n<k}} q_{n m}^{(1)} q_{k l}^{(1)} \sim\left(\frac{2}{\zeta_{2}}\right)^{2}\left(\frac{2}{\zeta_{2}}-1\right) \frac{\log t}{t^{2}}\left(-2 Z_{4}+Z_{3}\right) .
$$

As in the 3-point case we collect the contributions from (53), (55), and (56) giving

$$
\langle\sigma\rangle \sim 1+\frac{1}{9 \zeta_{2}^{2}} \frac{1}{t^{2}}\left(Z_{3}+Z_{4}+\frac{\log t}{d} \xi\left[-\left(\frac{3}{2 \zeta_{2}}+\frac{1}{2}\right) Z_{3}+\frac{4}{\zeta_{2}} Z_{4}\right]\right),
$$

and the dependence on $Z_{4}$ implies that

$$
\zeta_{4}=2(2-\xi)-\frac{4 \xi}{d}+O\left(\frac{1}{d^{3 / 2}}\right),
$$

in agreement with [5]. Eq. (57) yields further information by checking the contributions proportional to $Z_{3}$; the relative strength of the logarithmic terms is different from that obtained in the three point analysis, see (51). The reason is that $Z_{4}$ generates contributions proportional to $Z_{3}$ at first order, so that it is no longer an approximate zero mode when $d$ is finite, and the degeneracy between the 3-point and 4-point zero modes breaks down. It is not difficult to build the 4-point zero mode $\bar{Z}_{4}$ which continues correctly from infinite to finite dimensions as a linear combination of $Z_{3}$ and $Z_{4}$

$$
\bar{Z}_{4}=Z_{4}+\frac{4}{12-\xi} Z_{3}
$$

\subsection{The $t \rightarrow s$ transformation}

The previous analysis relied on calculations as a function of time, and the transformation to representation as a function of total scale was carried out by the simple substitution $t \rightarrow s^{\zeta_{2}}$. The purpose of this subsection is to show that corrections due to non-trivial dependence of $s$ on $t$ do not contribute terms of the order which was kept above, i.e. $O\left(1 / t^{2}\right)$ and $O\left(\log t /\left(d t^{2}\right)\right)$.

It should be emphasized that even when $d$ is very large, $s$ is not a monotonic function of $t$, and this is evident even in first order perturbation theory. However, the size of the time interval in which one is likely to find the same of value of $s$ becomes very small. In order to estimate the width of this interval, suppose $s(\bar{t})=\bar{s}$. We ask for which values of $\tau$ it is likely that $s(\bar{t}+\tau)=\bar{s}$ as well. We know that for small $\tau$

$$
s(\bar{t}+\tau) \sim \bar{s}+C \tau+\frac{B}{\sqrt{d}} \sqrt{\tau} \omega,
$$

where $\omega$ is a random variable with $O(1)$ variance, and $B$ and $C$ are some order 1 numbers. The deterministic part is monotonic and may be compensated by the fluctuating part to give a solution only if $\tau=O(1 / d)$. This is our estimate for the width of the multiple solution region. Replacing the earliest solution for $t(s)$ by some other arbitrary solution thus induces an $O(1 / d)$ error in $t$, which is too small to affect the results to the order that we keep.

In addition, up to $O(1 / d)$ we may assume that $s(t)$ is a one-to-one mapping, and may invert the relation to obtain $t(s) . s$ may be written (up to a constant multiplier) as

$$
s(t) \sim \sqrt{\frac{1}{N} \sum_{n m}\left(t+\tau_{n m}\right)^{2 / \zeta_{2}}\left(1+\frac{1}{\sqrt{d}} q_{n m}^{(1)}(t)+\cdots\right)} .
$$


Inverting to express $t$ as a function of $s$ gives

$$
t=t_{0}(s)\left(1-\frac{1}{t_{0}(s)} \frac{2}{\zeta_{2}} \sum_{n m} \tau_{n m}-\frac{1}{\sqrt{d}} \sum_{n m} q_{n m}^{(1)}\left(t_{0}(s)\right)\right)+\text { higher order terms }
$$

where $t_{0}=s^{\zeta_{2}}$. We see that to $O(1 / \sqrt{d}), t$ may indeed be considered as a single valued function of $s$ which is however randomly shifted with respect to the zero order estimate. We are now going to show that this correction does not contribute to the perturbative results either.

We substitute $t(s)$ in the asymptotic expression for $\sigma^{(0)}$ (see eq. 43, keeping the dependence on $\tau_{n m}$ implicit),

$\sigma^{(0)} \sim 1+\frac{F\left(\tau_{n m}\right)}{t^{2}} \sim 1+\frac{F\left(\tau_{n m}\right)}{t_{0}^{2}}\left(1+\frac{2}{t_{0}} \frac{2}{\zeta_{2}} \sum_{n m} \tau_{n m}+\frac{2}{\sqrt{d}} \sum_{n m} q_{n m}^{(1)}\left(t_{0}\right)+\frac{3}{d}\left(\sum_{n m} q_{n m}^{(1)}\left(t_{0}\right)\right)^{2}\right)$.

It follows after averaging that corrections to $t_{0}$ will contribute only terms of $O\left(1 / t_{0}^{3}\right)$ and $O\left(\log t_{0} /\left(d t_{0}^{4}\right)\right)$.

$\left\langle\sigma^{(1)}\right\rangle$, which vanishes when one takes $t=t_{0}$ as in the previous subsection, is nonzero when corrections to $t_{0}$ are taken into account. The leading non-zero terms are proportional to

$$
\frac{1}{\sqrt{d}} \sum_{n m} \frac{\tau_{n m} q_{n m}^{(1)}}{t_{0}} \sum_{k l} q_{k l}^{(1)}\left(t_{0}\right)
$$

whose expectation is of order $O\left(1 /\left(\sqrt{d} t_{0}\right)\right)$ and $O\left(d^{-3 / 2} \log t_{0} / t_{0}^{3}\right)$. Finally, it is clear that corrections to $t_{0}$ in $\left\langle\sigma^{(2)}\right\rangle$ cannot change the the leading logarithmic behavior. We conclude that to the order to which the above calculations we carried out it is safe to take $t=t_{0}=s^{\zeta_{2}}$.

\section{Conclusions and further applications}

In this paper we have presented a path oriented approach to the study of passive scalar advection. The main difference between this approach and other studies is that we do not need to consider the whole flow (and scalar) field, rather, an $N$-point correlation function is described via the evolution of $N$ Lagrangian trajectories. This allows us to study ordinary differential equations (albeit stochastic ones) instead of partial differential operators. The anomalous scaling is due to relaxation towards an invariant distribution of the instantaneous shapes. Since the relaxation rates are associated with excited states of an evolution operator these scaling exponents are not related to the normal scaling and dimensional reasoning cannot be applied.

The Lagrangian trajectories become nearly deterministic in very large dimensions (up to random solid body rotations). This property explains why the dynamics simplifies in this limit, and also serves as a starting point for an expansion. Using perturbation theory all the ingredients of the new anomalous scaling picture were demonstrated in an explicit and concrete manner, and perturbative corrections to the anomalous exponents were calculated.

In addition to the perturbative application it is possible to use the same concepts for numerical Monte-Carlo simulations. The main numerical task is to generate the random trajectories, which can be done using standard methods. However, in preliminary 
studies severe problems of convergence prevented us from obtaining precise results, and therefore the presentation of the numerical application is postponed [11, 12].

It would also be interesting to see how the other integrable limits of this model $(\xi \rightarrow 0$ and $\xi \rightarrow 2$ ) appear using the present ideas.

We would like to thank Uriel Frisch, Zeev Olami, Itamar Procaccia and Massimo Vergassola for discussions. O.G. thanks Krzystof Gawedzki and Itamar Procaccia, the organizers of the Turbulence Workshop in I.H.E.S in April 1997, where many of the ideas in this paper were developed. Most of this work was done while R.Z. was a CNRS post-doctoral fellow in the Laboratoire de Physique Statistique at the Ecole Normale Superieure (Paris). The Laboratoire de Physique Statistique is Laboratoire associé aux Universités Pierre et Marie Curie, Denis Diderot et au CNRS.

\section{References}

[1] U. Frisch, Turbulence, Cambridge University Press (1995).

[2] R. H. Kraichnan, Phys. Fluids 11945 (1968)

[3] R. H. Kraichnan, Phys. Rev. Lett., 721016 (1994)

[4] K. Gawȩdzki and A. Kupiainen, Phys. Rev. Lett., 75, 3834, (1995).

[5] M.Chertkov, G. Falkovich, I. Kolokolov and V. Lebedev, Phys. Rev. E 52 4924, (1995).

[6] O. Gat, V.S. L'vov, and I. Procaccia, Phys. Rev. E56, 406 (1997).

[7] A similar point of view is presented in D. Bernard, K. Gawedzki, A. Kupiainen, e-print cond-mat/9706035 (1997).

[8] See for example, I. T. Drummond, J. Fluid Mech. 123, 59 (1982), M. Chertkov, Phys Rev. E 55, 2722, (1997), P. M. Ginanneschi, e-print no. chao-dyn/9704017 (1997).

[9] B. Derrida, R. Zeitak, Phys. Rev. E. 54 pp 2513 (1996).

[10] R. H. Kraichnan, V. Yakhot and S. Chen, Phys. Rev. Lett., 75, 240 (1996).

[11] O. Gat, I. Procaccia, and R. Zeitak, unpublished (1997).

[12] The idea of using paths for passive scalar Monte-Carlo simulation was developed independently by U. Frisch and M. Vergassola. 